Case Report

\title{
Systemic Lupus Erythematosus with Multiple Autoimmune Disease Presented with Extensive Peripheral Gangrene
}

\author{
Tareq Z. Alzughayyar $\mathbb{D}^{1},{ }^{1}$ Jihad Samer Zalloum $\mathbb{D}^{1},{ }^{1}$ Mohammad N. Elqadi $\left(\mathbb{D},{ }^{1}\right.$ \\ Sadi A. Abukhalaf $\mathbb{D},{ }^{1}$ Fawzy M. Abunejma, ${ }^{2}$ Mohammed M. Y. Alkhanafsa, ${ }^{1}$ \\ Motasem H. M. Haif $\mathbb{D}^{1},{ }^{1}$ Firas Alqarajeh $\mathbb{D}^{1},{ }^{1}$ Mo'min R. Mesk $\mathbb{D}^{1},{ }^{1}$ and Rami A. Misk $\mathbb{D}^{3}$ \\ ${ }^{1}$ Al-Quds University, Faculty of Medicine, Jerusalem, State of Palestine \\ ${ }^{2}$ Pediatric Rheumatologist, Al-Ahli Hospital, Hebron, State of Palestine \\ ${ }^{3}$ An-Najah National University, Faculty of Medicine \& Health Sciences, Nablus, State of Palestine
}

Correspondence should be addressed to Tareq Z. Alzughayyar; tareq-20007@hotmail.com

Received 8 November 2019; Revised 15 January 2020; Accepted 17 February 2020; Published 10 March 2020

Academic Editor: George S. Habib

Copyright (C) 2020 Tareq Z. Alzughayyar et al. This is an open access article distributed under the Creative Commons Attribution License, which permits unrestricted use, distribution, and reproduction in any medium, provided the original work is properly cited.

\begin{abstract}
Systemic lupus erythematosus (SLE) is an autoimmune disease and can be associated with other autoimmune diseases. SLE usually presents with skin change and rarely presents with gangrene. SLE gangrene usually involves the digits of upper extremities. We report the first case of SLE associated with an extremely rare constellation of neuromyelitis Optica (NMO) and diabetes mellitus type 1, presented with a rare form of the SLE gangrene which involves bilateral lower extremities up to midlegs, a case that has not yet been reported in the literature. Although SLE gangrene may respond to immunosuppressants, it has a high risk of complications that can end up with amputations.
\end{abstract}

\section{Introduction}

Systemic lupus erythematosus (SLE) is an autoimmune disorder leading to inflammation and tissue damage involving multiple organ systems and is more common in females with a $3: 1$ ratio [1]. SLE can present in a variety of clinical presentations. Skin manifestations are one of the most common presenting symptoms. However, gangrene is an exceptionally rare form of SLE cutaneous manifestations, accounting for 1.4\% of SLE patients [2]. SLE gangrene most often affects the upper extremities and involves no more than the digits [3]. Pathophysiology underlying SLE gangrene includes vasospasm, thromboembolism, and vasculitis [4]. SLE vasculitis is uncommon and is usually small or medium-sized vessel vasculitis (9.7\% and $1.6 \%$, respectively), though large-vessel vasculitis are very unusual [5]. SLE is rarely associated with the autoimmune demyelinating Neuromyelitis Optica (NMO) which predominantly involves optic nerves and spinal cord. [6]. Herein, we report a 10-year-old female with multiple autoimmune diseases (SLE, NMO, and type $1 \mathrm{DM}$ ) presented with bilateral lower limb bluish cyanotic discoloration that rapidly developed into gangrene, necessitating bilateral below-knee amputations. This is the first case reported with such complex constellation in the literature as far as our knowledge and available data.

\section{Case Presentation}

A 10-year-old girl with a known history of systemic lupus erythematosus presented to the emergency department with acute painful bluish discoloration involving bilateral feet of rapid onset beginning 1 week ago (Figure 1).

Upon admission and during the physical examination, the patient had blue to red (violet) discoloration mainly involving feet, with the involvement of the hands and face, resembling maculopapular rash, and had active polyarthritis in the joints of the PIPs, MCPs, ankles, and right knee. 
Lesions of the legs were bilateral below-ankle cyanotic changes with more severe and of wider spread in the right foot. No ulceration or gangrenous changes were noted. Dorsal pedis and posterior tibial pulses were sluggish and weak.

The patient was admitted, and Laboratory investigations were conducted (Tables 1 and 2). Blood and urine cultures were negative. Chest $\mathrm{x}$-ray, echocardiography, foot $\mathrm{x}$-ray, and abdominal sonography all were normal. Doppler ultrasound and CT angiography were positive (Figures 2 and 3).

IV Methylprednisone, IV Heparin infusion, IVIG, Methotrexate, and Antihypertensive drugs (carvedilol and Amlodipine) were given.

After treatment, proteinuria, c3, and c4 normalized, where ESR did not improve and remained around 80-110.

During admission, pedal lesions progressed into dark purple discoloration and spread $4 \mathrm{~cm}$ above the ankle bilaterally (Figure 4). An increase in pedal pain and hypothermia were also reported. Examination showed absent pulse in distal popliteal, tibial arteries, and dorsal pedis arteries, which appears as ascending compromise of blood flow. Unfortunately, bilateral below-knee amputation warranted due to loss of viability.

In a previous visit to the Emergency department (days after onset, before admission), the patient reported the first symptom as sudden blue to red discoloration in the tip of the toes that rapidly spread to involve proximal portions of feet, associated with pain and hypothermia and has been treated as an outpatient with low-dose steroid, Azathioprine, MTX, Vitamin D, and Hydroxychloroquine.

Our patient was not only diagnosed with SLE at the age of nine according to SLICC (Systemic Lupus International Collaborating Clinics) and ACR criteria (Table 2) but also was known to have NMO from her first year of life and DM1 at the age of 7; the 10-year-old girl was paraplegic, blind, and in poor glycaemic control when presented to us, complicated by bilateral peripheral ischemia, which was confirmed by CT angiography (Figures 2 and 3 ) and was rapidly progressing to gangrene in spite of treatment.

\section{Discussion}

We report this case for its unusual constellation of different autoimmune pathologies. As far as we know and based on available data, there are no reports in the literature of SLE occurring in a child with $\mathrm{NMO}$ and type $1 \mathrm{DM}$, complicated by peripheral gangrene.

SLE is an autoimmune multisystemic disease, linked to considerable morbidity and mortality but no known etiology [7]. Cutaneous manifestations are valuable in regards to diagnosis and prognosis due to the fact of 7 out of 11 ARA criteria for SLE are cutaneous [8]. Digital ischemia, digital ulcer, and gangrenous lesions, though rare, it is previously reported in SLE.

Peripheral gangrene in SLE is very rare, especially if it involves large and medium-sized arteries. Occurring in about $1 \%$ of SLE patients, and most often affects the upper

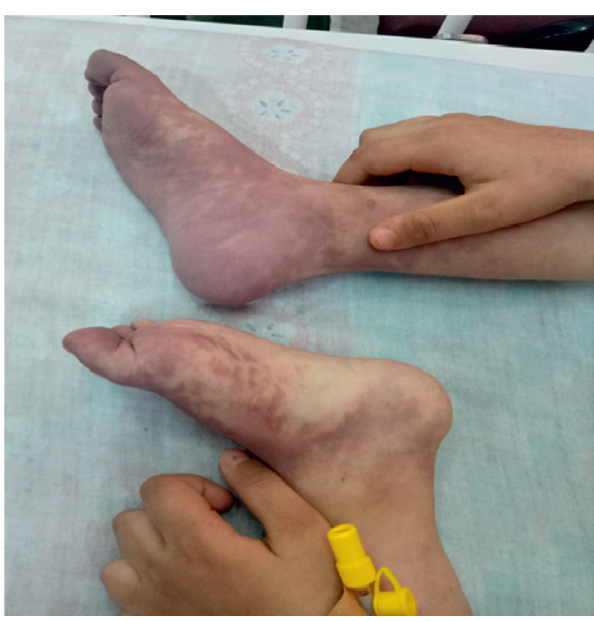

Figure 1: Bilateral bluish feet at admission.

TABLE 1: Routine laboratory tests.

\begin{tabular}{lc}
\hline Lap test & Value \\
\hline ECR & $100 \mathrm{~mm} / \mathrm{h}$ \\
CRP & $8 \mathrm{mg} / \mathrm{l}$ \\
HbA1c & $9.6 \%$ \\
Random blood sugar & $200 \mathrm{mg} / \mathrm{dl}$ \\
BUN & $16 \mathrm{mg} / \mathrm{dl}$ \\
Creatinine & $1 \mathrm{mg} / \mathrm{dl}$ \\
Aspartate aminotransferase (AST) & $22 \mathrm{U} / \mathrm{L}$ \\
Alanine aminotransferase (ALT) & $18 \mathrm{U} / \mathrm{L}$ \\
\hline
\end{tabular}

extremities [9-11], it is considered a severe complication that generally leads to amputation [12].

Risks factors for SLE peripheral gangrene include: long disease duration ( $\geq 4$ years), Raynaud's phenomenon, and elevated serum C-reactive protein (CRP) [11]. Moreover, Anti-RNP may aid the development of digital gangrene as found to have an association with Raynaud phenomenon and APS $[8,13,14]$.

Causes of SLE peripheral gangrene are unknown and multifactorial, and the underlying mechanism is complex and diverse, though can be summarized as follows: (1) vasculitis and infectious disorders that could cause vasculitis, (2) rheumatological disorders, (3) mechanical and obstructive disorder, (4) premature atherosclerosis, (5) vasospasm, (6) overlap syndrome, and (7) hypercoagulabilitythrombosis related to antiphospholipid (APL) antibodies or embolus originating from the heart secondary to Libmansack endocarditis, all of which may contribute to the development of gangrene [12, 15-22].

Inherited classical complement deficiencies can be associated with SLE as it shares some common immunological and clinical features, especially early age onset of cutaneous involvement. However, as reported in the literature, the presence of positive anti-dsDNA antibodies, negative anticardiolipin antibodies, and normalization of complement level after medical treatment does not support complement components deficiency $[23,24]$. 
TABLe 2: New ACR and EULAR criteria scoring for our case.

\begin{tabular}{|c|c|c|c|c|}
\hline Domain & Criteria & Points & Patient value & Patient score \\
\hline \multicolumn{5}{|l|}{ ANA $=1: 120$} \\
\hline \multicolumn{5}{|c|}{ EULAR/ACR clinical domains and criteria for SLE } \\
\hline \multirow[t]{2}{*}{ Constitutional } & Fever & 2 & Positive & 2 \\
\hline & Leukopenia & 3 & WBC: 10,000 & 0 \\
\hline \multirow[t]{3}{*}{ Hematologic } & Thrombocytopenia & 4 & PLT: 233,000 & 0 \\
\hline & Autoimmune hemolysis & 4 & $\mathrm{Hb}: 10 \mathrm{~g} / \mathrm{dl}$ & 0 \\
\hline & Delirium & 2 & & 0 \\
\hline \multirow{3}{*}{ Neuropsychiatric } & Psychosis & 3 & Negative & 0 \\
\hline & Seizure & 5 & & 0 \\
\hline & Nonscarring alopecia & 2 & & 0 \\
\hline \multirow{3}{*}{ Mucocutaneous } & Oral ulcers & 2 & & 0 \\
\hline & Subacute cutaneous or discoid lupus & 4 & Positive only for acute cutaneous lupus & 0 \\
\hline & Acute cutaneous lupus & 6 & & 6 \\
\hline \multirow{2}{*}{ Serosal } & Pleural or pericardial effusion & 5 & Negative & 0 \\
\hline & Acute pericarditis & 6 & Negative & 0 \\
\hline \multirow[t]{2}{*}{ Musculoskeletal } & Joint involvement & 6 & Positive & 6 \\
\hline & Proteinuria $>0.5 \mathrm{~g} / 24 \mathrm{~h}$ & 4 & $0.51 \mathrm{~g} / 24 \mathrm{hr}$ & 4 \\
\hline \multirow[t]{2}{*}{ Renal } & Renal biopsy class II or V lupus nephritis & 8 & negative & 0 \\
\hline & Renal biopsy class III or IV lupus nephritis & 10 & negative & 0 \\
\hline
\end{tabular}

EULAR/ACR immunologic domains and criteria for SLE

\begin{tabular}{|c|c|c|c|c|}
\hline $\begin{array}{l}\text { Anti-phospholipid } \\
\text { antibodies }\end{array}$ & $\begin{array}{c}\text { Anti-cardiolipin antibodies or } \\
\text { Anti- } \beta 2 \mathrm{GP} 1 \text { antibodies or } \\
\text { Lupus anticoagulant }\end{array}$ & 2 & $\begin{array}{l}\text { Anti-cardiolipin IgG: } 0.6 \mu / \mathrm{ml} \\
\text { Anti- } \beta 2 \mathrm{GP1} \text { IgM: } 2.2 \mu / \mathrm{ml} \\
\text { Anti- } \beta 2 \mathrm{GP1} \text { IgG: } 0.3 \mu / \mathrm{ml} \text {. } \\
\text { Lupus anticoagulants PTT: } \\
30 \text { seconds }\end{array}$ & 0 \\
\hline Complement proteins & $\begin{array}{c}\text { Low C3 or low C4 } \\
\text { Low C3 and low C4 }\end{array}$ & $\begin{array}{l}3 \\
4\end{array}$ & $\begin{array}{c}\mathrm{C} 3=33 \\
\mathrm{C} 4=5\end{array}$ & 4 \\
\hline SLE-specific antibodies & $\begin{array}{c}\text { Anti-dsDNA antibody or } \\
\text { Anti-Smith antibody }\end{array}$ & 6 & $\begin{array}{c}\text { Positive Anti-dsDNA: } \\
\text { IgM }=200 \\
\operatorname{IgG}=70\end{array}$ & 6 \\
\hline
\end{tabular}

Total patient score $=28$

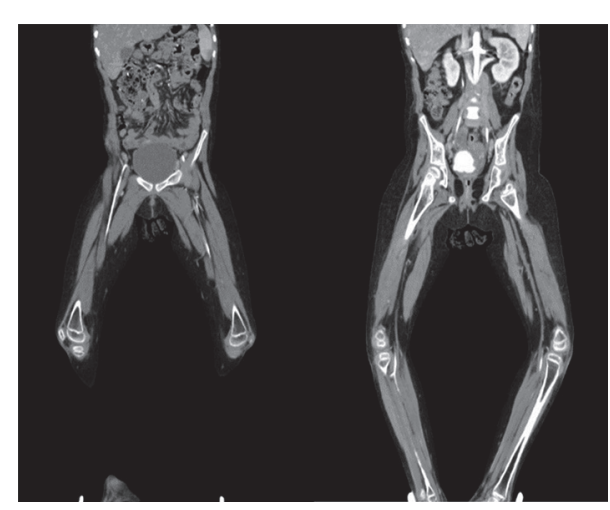

FIGURE 2: Contrast (white) shows weak blood flow in iliac, femoral, and popliteal arteries.

$\mathrm{DM}$ is also a possible cause of peripheral gangrene; though our patient had DM for 2 and half years, it is unlikely to be the causative agent in our case because of bilateral symmetrical involvement of both lower limbs and the very acute onset and deterioration within days.

Of the mentioned causes, vasculitis and APS are repeatedly linked with gangrene in the setting of SLE. Most of the reported cases indicate the presence of antiphospholipid antibodies, indicating that APS could be the largest contributor.

APS is significantly associated with gangrene in SLE patients (3.3-7.5\%) [13, 25]. Increased ANA and Anti- $\beta 2$ glycoprotein I autoantibodies were noted in some cases of peripheral gangrene $[13,26,27]$.

Vasculitis in SLE occurs almost exclusively in small vessels. The primary pathology is leukocytoclastic vasculitis. Medium- and large-vessel vasculitis in association with SLE is unusual [16].

Recognizing the underlying cause of peripheral gangrene in SLE is aided by histopathology, imaging modalities, and serological markers.

Histopathology can reveille the underlying cause, vasculitis vs. thrombosis, or mixture of both (secondary vasculitis in APS) $[9,28]$. Vasculitis signs can be demonstrated in the less-invasive modality CT angiography, namely, wall thickening, irregularity, and luminal narrowing. TEE detects vegetations of Libman sac endocarditis and can also aid diagnosis [29]. The serological marker can put light on the possibility of APS when antiphospholipid (APL) antibody is present.

In a nutshell, the diagnosis of underlying pathology is much of importance and can guide us into more clear management, vasculitis vs. hypercoagulability treatment. 


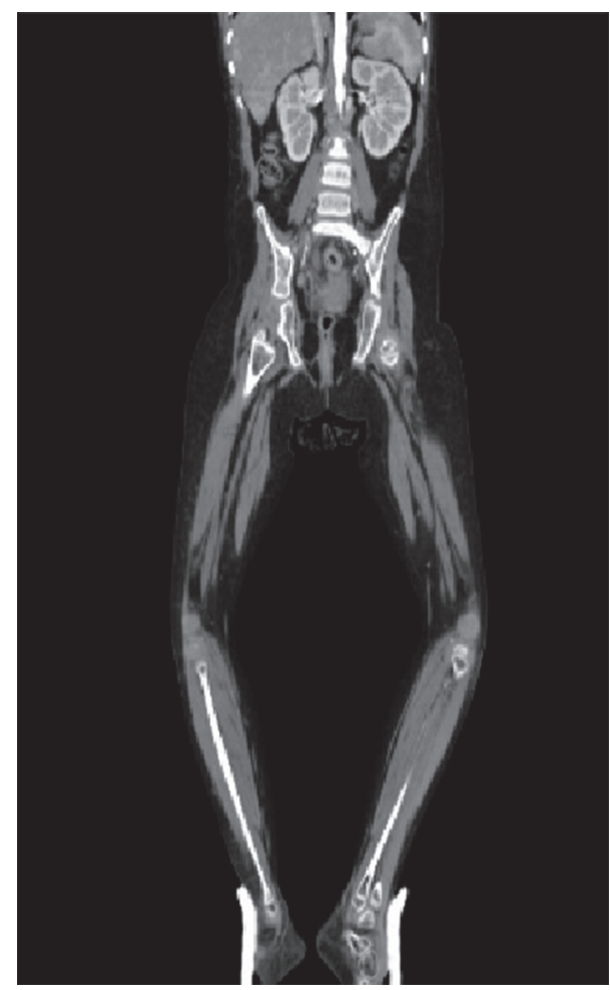

FIGURE 3: No contrast visible in distal popliteal and tibial arteries (no blood flow).

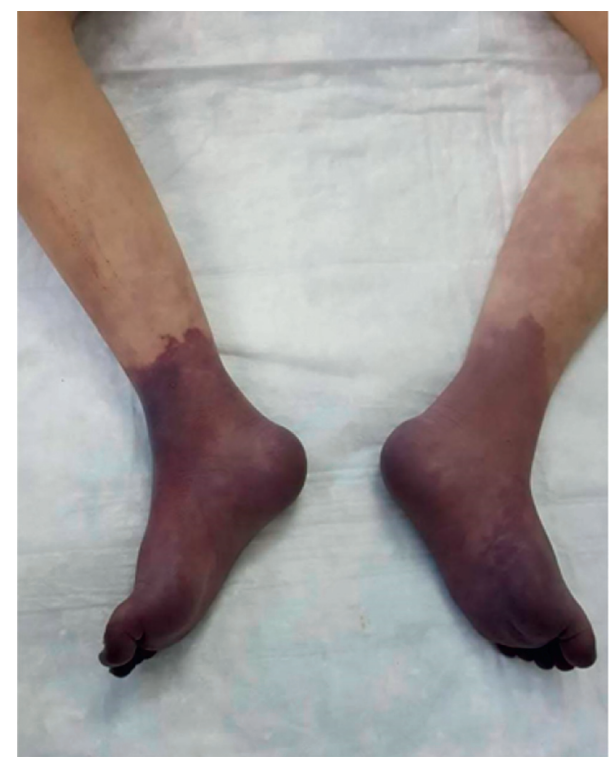

FIGURE 4: Gangrenous rash involves the whole feet and the lower part of the legs bilaterally.

Our patient had negative antiphospholipid (lupus anticoagulants, Anticardiolipin, and anti B2-glycoprotein) antibodies twice 6 months apart and no previous hx of any thrombotic event, CVA, or peripheral vascular events, and imaging wise there was no evidence of thrombosis; thrombophilia profile (protein C, S and Leiden v) was normal when she was investigated due to NMO and paraplegia, which makes thrombosis less likely to be the cause of this gangrene.

Treatment of SLE gangrene is generally divided into two classes:

(1) Anticoagulation: heparin and warfarin for APS/LAC.

(2) Immunosuppression: corticosteroid and immunosuppressive drugs for vasculitis [30]. Rituximab, lowdose epoprostenol, mycophenolate, and cyclophosphamide can also be used $[7,8,31]$.

Liu et al. study suggested that early treatment with an aggressive corticosteroid, immunosuppressive drugs, lipid lowering agents, and anticoagulation therapies in patients complicated with antiphospholipid syndrome may prevent gangrene from deterioration, decrease the hazard of amputation, and improve prognosis in patients with SLE $[11,32]$.

Surgical resection is reserved for severe cases as our patient, who despite the combination therapy, deteriorated rapidly. However, many patients resolve without any complications, while some have auto amputations [9].

Concerning prognosis, some medical literature observed an unusual phenomenon that after gangrene, many patients entered a state of clinical remission and for unknown reasons could be a good prognostic sign in SLE. Or due to the fact that gangrene is such an alarming sign, early diagnosis and aggressive treatment could recover the balance of the immune system, avoiding future relapses $[9,10]$. Lacking data on cases that progressed to death or severely deteriorated could falsely present this fact to us, and though controversial, it was worth mentioning [9].

Prevention included control of traditional and diseaserelated risk factors [33]. Also, patients of SLE vasculitis should avoid cold exposure due to the risk of gangrene [34].

In conclusion, despite their young age, SLE patients are at increased risk of developing peripheral ischemic gangrene, and diagnosis of underlying pathology is much of importance and can guide us into more clear management. Early and aggressive corticosteroid treatment prevented gangrene from progression and improved prognosis.

\section{Consent}

The patient and family's consent was obtained.

\section{Conflicts of Interest}

The authors declare that there are no conflicts of interest regarding the publication of this article.

\section{Authors' Contributions}

Tareq Z. Alzughayyar, SadiA. Abukhalaf, Fawzy M. Abuniejma, and Firas Alqarajeh were involved in the study concept or design. Data collection was performed by Mohammad N. Elqadi, Mohammed M. Y. Alkhanafsa, Motasem H. M. Haif, and Mo'min R. Mesk. Tareq Z. Alzughayyar, Jihad Samer Zalloum, and Rami A. Misk wrote the paper. 


\section{References}

[1] R. G. Lahita, "The role of sex hormones in systemic lupus erythematosus," Current Opinion in Rheumatology, vol. 11, no. 5, pp. 352-356, 1999.

[2] R. C. Jeffery, C. B. Narshi, and D. A. Isenberg, "Prevalence, serological features, response to treatment and outcome of critical peripheral ischaemia in a cohort of lupus patients," Rheumatology, vol. 47, no. 9, pp. 1379-1383, 2008.

[3] V. B. Shetty, S. Rao, P. N. Krishnamurthy, and V. U. Shenoy, "Peripheral gangrene during infancy: a rare presentation of systemic lupus erythematosus," Archives of Disease in Childhood, vol. 85, no. 4, pp. 335-336, 2001.

[4] A. H. Kao, J. M. Sabatine, and S. Manzi, "Update on vascular disease in systemic lupus erythematosus," Current Opinion in Rheumatology, vol. 15, no. 5, pp. 519-527, 2003.

[5] M. Ramos-Casals, N. Nardi, M. Lagrutta et al., "Vasculitis in systemic lupus erythematosus," Medicine, vol. 85, no. 2, pp. 95-104, 2006.

[6] A. Iyer, L. Elsone, R. Appleton, and A. Jacob, "A review of the current literature and a guide to the early diagnosis of autoimmune disorders associated with neuromyelitis optica," Autoimmunity, vol. 47, no. 3, pp. 154-161, 2014.

[7] O. Küçükşahin, N. Düzgün, A. K. Okoh, and E. Kulahçioglu, "Response to rituximab in a case of lupus associated digital ischemia," Case Reports in Rheumatology 2014, vol. 2014, Article ID 763608, 4 pages, 2014.

[8] N. Kapadia and T. S. Haroon, "Cutaneous manifestations of systemic lupus erythematosus: study from Lahore, Pakistan," International Journal of Dermatology, vol. 35, no. 6, pp. 408-409, 1996.

[9] M. C. B. T. da Rocha, M. J. P. Vilar, E. A. M. Freire, and M. B. Santiago, "Arterial occlusion in systemic lupus erythematosus: a good prognostic sign?” Clinical Rheumatology, vol. 24, no. 6, pp. 602-605, 2005.

[10] P. Dieppe, F. A. Wollheim, and H. Ralph Schumacher, "Clinical manifestations of systemic lupus erythematosus. Computer analysis of 520 cases: dubois EL, Tuffanelli DL," Classic Papers in Rheumatology, CRC Press, Boca Raton, USA, pp. 178-179, 2001.

[11] A. Liu, W. Zhang, X. Tian, X. Zhang, F. Zhang, and X. Zeng, "Prevalence, risk factors and outcome of digital gangrene in 2684 lupus patients," Lupus, vol. 18, no. 12, pp. 1112-1118, 2009.

[12] M. A. Alghamdi, "Digital gangrene as the initial presentation of systemic lupus erythematosus without secondary antiphospholipid syndrome: case report," Zagazig University Medical Journal, vol. 21, p. 3, 2015.

[13] R. A. Asherson, "The antiphospholipid antibody syndrome: diagnosis, skin manifestations and current therapy," Clinical and Experimental Rheumatology, vol. 24, no. 1, p. S46, 2006.

[14] R. W. Hoffman, G. C. Sharp, and S. L. Deutscher, "Analysis of anti-u1 RNA antibodies in patients with connective tissue disease," Arthritis \& Rheumatism, vol. 38, no. 12, pp. 1837-1844, 1995.

[15] R. Kashyap, "Symmetrical peripheral gangrene due to viral gastroenteritis," JAPI, vol. 52, pp. 500-501, 2004.

[16] C. Varma, S. Aroor, S. C. Mundkur, and K. Annamalai, "Kawasaki disease with peripheral gangrene and autoamputation-an extremely rare complication: a case report," Our Dermatology Online, vol. 3, no. 3, pp. 210-211, 2012.

[17] D. Singh and R. Rai., "Hypernatremic dehydration leading to peripheral gangrene," Indian Pediatrics, vol. 45, no. 6, p. 513, 2008.
[18] J. Raghunandan, K. Rajeshwari, A. P. Dubey, and T. Singh, "Peripheral gangrene in an 18-month-old boy with Plasmodium vivaxmalaria," Paediatrics and International Child Health, vol. 32, no. 3, pp. 164-166, 2012.

[19] S. Avasthi and R. K. Tandon, "Peripheral gangrene in a child as a result of primary thrombocytosis," Internet Journal of Medical Update-EJOURNAL, vol. 7, no. 1, 2012.

[20] A. P. Dubey, S. Sudha, and A. Parakh, "Peripheral gangrene: an uncommon manifestation of disseminated tuberculosis," Indian Pediatrics, vol. 43, no. 3, p. 255, 2006.

[21] M. Malina, A. Gulati, A. Bagga, M. A. Majid, E. Simkova, and F. Schaefer, "Peripheral gangrene in children with atypical hemolytic uremic syndrome," Pediatrics, vol. 131, no. 1, pp. e331-e335, 2013.

[22] R. A. Asherson, R. Cervera, and Y. Shoenfeld, "Peripheral vascular occlusions leading to gangrene and amputations in antiphospholipid antibody positive patients," Annals of the New York Academy of Sciences, vol. 1108, no. 1, pp. 515-529, 2007.

[23] M. Kallel-Sellami, L. Laadhar, Y. Zerzeri, and S. Makni, "Complement deficiency and systemic lupus erythematosus: consensus and dilemma," Expert Review of Clinical Immunology, vol. 4, no. 5, pp. 629-637, 2008.

[24] R. M. Ekinci, “Autoimmune manifestations in heterozygote type I complement 2 deficiency: a child eventually diagnosed with systemic lupus erythematosus," Archives of Rheumatology, vol. 34, no. 1, pp. 96-99, 2019.

[25] G. Knarik, "Clinical characteristics of patients with systemic lupus erythematosus with anticardiolipin antibody," APLAR Journal of Rheumatology, vol. 9, no. 1, pp. A21-278, 2006.

[26] S. Paira, "Extensive cutaneous necrosis associated with anticardiolipin antibodies," The Journal of Rheumatology, vol. 26, no. 5, pp. 1197-1200, 1999.

[27] Z. Vasugi and D. Danda, "Systemic lupus erythematosis with antiphospholipid antibody syndrome: a mimic of Buerger's disease," Journal of Postgraduate Medicine, vol. 52, no. 2, p. 132, 2006.

[28] M. S. Klein-Gitelman and M. L. Miller, "Systemic lupus erythematosus," The Indian Journal of Pediatrics, vol. 63, no. 4, pp. 485-500, 1996.

[29] N. Kumar, N. Choudhary, G. Agarwal, Y. Rizvi, B. Kaul, and R. Ahlawat, "Extensive medium-vessel vasculitis with SLE," JCR: Journal of Clinical Rheumatology, vol. 13, no. 3, pp. 140-142, 2007.

[30] H. Amital, "Leg amputation-possible therapeutic role in catastrophic anti-phospholipid syndrome (CAPS)," Lupus, vol. 11, p. 554, 2002.

[31] A. R. Hong, D. P. Croitoru, L. T. Nguyen, J.-M. Laberge, Y. Homsy, and G. H. Kiruluta, "Congenital urethral fistula with normal anus: a report of two cases," Journal of Pediatric Surgery, vol. 27, no. 10, pp. 1278-1280, 1992.

[32] E. Vocks, M. Welcker, and J. Ring, "Digital gangrene: a rare skin symptom in systemic lupus erythematosus," Journal of the European Academy of Dermatology and Venereology, vol. 14, no. 5, pp. 419-421, 2000.

[33] S. M. A. Toloza, A. G. Uribe, G. McGwin et al., "Systemic lupus erythematosus in a multiethnic US cohort (LUMINA): XXIII. Baseline predictors of vascular events," Arthritis \& Rheumatism, vol. 50, no. 12, pp. 3947-3957, 2004.

[34] N. Wong, V.-Y. Ng, S. Ibrahim, M. Slessarev, and V. Chandran, "Lupus-the cold, hard facts," Lupus, vol. 23, no. 8, pp. 837-839, 2014. 